\title{
What questions are most important to pancreatic cancer patients soon after diagnosis? A multicenter survey
}

\author{
Lisa J. M. Ronde-Schoone ${ }^{1}$, Chulja J. Pek ${ }^{2}$, Rutger-Jan Swijnenburg ${ }^{3}$ and Arwen H. Pieterse $4^{4^{*}}$ (D)
}

\begin{abstract}
Background: Pancreatic cancer has a poor prognosis. Patients might be better able to cope with their disease when the information is discussed that they consider most important. We investigated what questions pancreatic patients consider most important to address in the first weeks after diagnosis.

Methods: We built a survey listing 84 questions and for each asked how important (range, 1-7) individuals who had received a certain or likely diagnosis of pancreatic cancer considered it that the question was addressed soon after diagnosis; patients who completed the survey 1 year or more after diagnosis were excluded. Mean perceived importance scores were used to rank order the questions in terms of importance.

Results: Forty-seven pancreatic cancer patients participated. The participants considered receiving an answer to a median of 53 (range, 21-83) questions as important (score $=6$ ) or very important (score $=7$ ). The number was not significantly related to gender, age, education, or time since diagnosis. For 42/84 questions, average score was $\geq 6.0$. Topics considered most important included diagnosis, likelihood of cure, treatment options, harms and procedures, prognosis if the disease were left untreated, and quality of life. For $67 / 84$ questions, $\geq 1$ participants indicated that answering the question should be avoided (Md $=1$ participant, range, 1-5) and for $77 / 84$ questions that it was not applicable ( $M d=3.5$ participants, range, 1-30).

Conclusions: Pancreatic cancer patients consider a wide range of questions important to address after diagnosis, including those on sensitive topics. Doctors need to carefully dose information provision to avoid overloading patients. The findings can help to guide doctors and other information resources to provide relevant information to pancreatic cancer patients.
\end{abstract}

Keywords: Pancreatic cancer, Information preferences, Question topics, Survey

\section{Background}

Worldwide 338,000 people are diagnosed with pancreatic cancer each year, and it is predicted to become the second most common cause of cancer-related death in 2030 [1]. The disease has a very poor prognosis; approximately six out of 100 patients survive 5 years after diagnosis [2]. Early detection of pancreatic cancer is challenging because patients are often asymptomatic or experience non-specific symptoms until late stage disease. When a pancreatic mass is detected using clinical

\footnotetext{
*Correspondence: Pieterse@lumc.nl

${ }^{4}$ Department of Medical Decision Making, Leiden University Medical Centre, P.O. Box 9600, Leiden 2300 RC, the Netherlands

Full list of author information is available at the end of the article
}

imaging modalities such as ultrasound and/or CT scan, it is generally unclear whether a malignancy is causing the mass. Usually, biopsies via endoscopic ultrasound are needed, or surgery is performed while the diagnosis is still unclear. At primary diagnosis, up to $85 \%$ of patients present with advanced unresectable disease, due to locoregional spread and metastases. For these patients, only palliative treatment can be offered. For 15\% of patients surgery is possible at diagnosis, which is the only therapy that may be curative $[3,4]$. However, even after surgery survival rates are low, and almost all patients die from their disease within 7 years [4].

Since so little can be done to help pancreatic cancer patients in terms of cure and the diagnostic process is 
often long and uncertain, it becomes increasingly important to seek other ways of helping patients to cope with their (suspected) disease. One of these ways is to tailor information provision, which is of particular importance in the period after diagnosis [5]. At that time, patients learn more about the disease and their prognosis, and treatment decisions are made. Evidence suggests that adequate information can improve cancer patients' well- being [6], give patients a feeling of control [7], and help patients to cope with their disease $[8,9]$. Information can also reduce anxiety and depression [10], improve treatment compliance, create more realistic expectations about the treatment, promote self-care and participation in one's care, and generate feelings of safety and security [7]. However, research has shown that the information that cancer care professionals provide to cancer patients often does not match the patients' information needs [11]. Obviously, providing the adequate amount or type of information is challenging. Some patients desire a lot of information, while others feel overwhelmed by the amount of information they receive [6]. Also, cancer patients differ in the type of information they want to hear $[6,8,12]$. We performed a multicentre survey aimed at identifying what questions are most important for pancreatic cancer patients to be answered in the first period after diagnosis.

\section{Methods}

\section{Participants}

Any individual who recently received a certain or likely diagnosis of pancreatic cancer was eligible for the study.

\section{Procedure}

Recruitment took place between April 2013 and May 2014. Patients were recruited through the Internet or at their visit at the hospital. A call with information about the study was placed on the websites of Dutch foundations which aim to address needs of patients with pancreatic cancer or digestive diseases (Lisa Waller Hayes foundation, Dutch Digestive Diseases Foundation, Foundation for Patients with Alimentary Canal Cancer). The call included a link to an online survey as well as contact information to receive the paper version and a postagepaid return envelope. Furthermore, surgeons, gastroenterologists, physician assistants and nurse practitioners from three academic and three non-academic Dutch hospitals recruited patients, at the time of their outpatient appointment or when admitted to the hospital. The health care professionals gave eligible patients a flyer with information about the study including the link to the online survey, again with the option to complete it on paper. The participants provided informed consent before completing the survey in one of two ways. They were either asked to sign an informed consent form if completing the survey on paper or were asked for informed consent on the first screen of the online survey and were only directed to the actual survey if they responded positively. The Leiden University Medical Center Medical ethics committee approved the study.

\section{Survey development}

To generate a list of questions which could be important to patients with pancreatic cancer in the first weeks after their diagnosis, nine patients (four male, five female) were interviewed. Eight patients received the diagnosis pancreatic cancer and one patient first was suspected to have pancreatic cancer, and turned out to have bile duct cancer. The interviewer (LRS) asked patients which questions were important to them in the first weeks after diagnosis. To support patients' thinking and recollection, the interviewer asked them to name any question they could think of in the following categories [5]: disease, treatments, physical, and psychosocial issues. The questions obtained from these nine interviews were included if they seemed general enough to be relevant to more patients as well as specific enough to the disease and treatment options at stake. This was determined by consensus among the authors (LRS, RJS, AP). Question topics most often mentioned included questions about the treatment procedure, treatment harms, caregiver support, and what diet is most suitable for pancreatic cancer patients. The questions obtained from the interviews were combined with questions that could possibly be relevant to patients with pancreatic cancer in the first weeks after diagnosis, based on studies on information needs in prostate cancer patients $[6,13]$. This resulted in a total of 84 questions.

\section{Final survey}

The final survey consisted of two parts. It started with questions about the participants' personal situation, including age, gender, level of education, time since diagnosis, and treatment or treatments received or about to be started in the upcoming month.

Next, patients were asked to think back to the first weeks after diagnosis and to indicate how important it was to them, at that time, that certain questions were answered. The participants were asked to rate the importance of each of the 84 questions on a seven-point Likerttype scale, ranging from 1 ,"totally not important", through 4 "not unimportant/not important" to 7, "very important". The seven-point Likert-type scale was chosen to enable patients to distinguish the importance of different questions. Each point on the response scale was labelled. If the participants deemed it appropriate, they could instead tick one of two boxes: "avoid answer", to indicate that a question should not be answered, or "not applicable", to indicate that a question is not deemed relevant to pancreatic cancer soon after diagnosis. 
To structure the large amount of questions, they were arranged into the following categories [5]: disease, treatments, physical issues, and psychosocial issues. Within the category "disease", questions about the nature of the disease were followed by questions about disease progress and prognosis. Within the category "treatments", questions were ordered as following: questions about a) the choice whether or not to treat the disease, b) treatment procedures, c) treatment harms, and d) treatment results.

\section{Statistical analyses}

Descriptive statistics were used to present the number of questions that the participants rated as important or very important and the perceived importance of questions. We assessed whether the number of questions a participant rated as important or very important was significantly related to gender, age, education, or number of days since diagnosis using median tests and spearman correlations. We used the mean perceived importance score to rank order the questions in terms of importance. In case of equal mean importance scores, questions with a smaller standard deviation were considered as more important, assuming that there was comparatively more agreement on their importance among the participants. The participants who indicated that a question was not applicable or that it should be avoided, are shown separately. We compared the top 10 of most important questions, again rank ordered according to their mean score and standard deviation, as a function of time using a median split to separate the patients who had completed the questionnaire earlier (i.e., within 46 days of diagnosis) versus later (i.e., after 46 days). SPSS 22 was used to analyse the data, significance was tested two-tailed at $\alpha=0.05$.

\section{Results \\ Participants}

Forty-seven participants were included of whom 31 were recruited through an hospital and 12 were included through the Internet. For four patients it is unknown how they came across the online survey. The patients had been diagnosed between 0 and 206 days before completing the survey (Table 1). One patient completed the survey on the day of diagnosis and seven others within 10 days after diagnosis. Three patients received their diagnosis more than 6 months before completing the survey. About half of the participants were male. Most participants had had surgery with the intent to remove the tumour. Overall, there were very few missing responses to the survey (two participants omitted 1/84 question, one participant omitted 10/84 questions).
Table 1 The participants' socio-demographic and disease-related characteristics $(N=47)$

\begin{tabular}{ll}
\hline Patient characteristic & $\mathrm{N}(\%)$ \\
\hline Male gender & $26(55)$ \\
Mean age (range) & $58.9 \pm 11.4(27-84)$ \\
Education & \\
Low & $8(17)$ \\
Intermediate & $17(36)$ \\
High & $18(38)$ \\
Median time since (likely) diagnosis, in days (range) & $46 \pm 58(0-206)$ \\
Treatment received or about to receive in upcoming month \\
Surgery & $34(72)$ \\
Chemotherapy & $12(26)$ \\
Stent implantation & $11(23)$ \\
Post-surgery enzymes & $10(21)$ \\
Pain management & $10(21)$ \\
Laparoscopy & $8(17)$ \\
Drain in the liver & $2(4)$ \\
Treatment for excessive fluids & $2(4)$ \\
Radiotherapy & $1(2)$ \\
No treatment received or planned & $5(10)$
\end{tabular}

${ }^{a}$ Numbers do not add up to $N=47$ because of missing data

\section{Perceived importance of questions}

The participants rated a median of 53/84 (63\%, range, 21-83) questions as important or very important (score $\geq 6$ ). Gender, age, educational level, and time since diagnosis did not significantly influence the number of questions judged to be (very) important.

With regard to how important individual questions were rated, $42 / 84$ questions were considered to be important ( $50 \%$, mean score $\geq 6$, Table 2 ). The top three of most important questions to be addressed asked about the presence of metastases, if surgery was still possible, and what the likelihood of cure is. The top 10 of questions that were perceived as most important in participants who completed the survey earlier versus later after diagnosis both included the five questions rated as most important in the full sample, and each of the remaining top-10 questions was rated overall with a mean important score of 6.41 or higher (Table 2). Generally, the questions that the participants rated as most important related to disease (diagnosis, natural course, cure), treatment (options, benefits, harms, procedures), what happens if the disease is not treated, as well as pain, fatigue, and diet. Eleven of 84 (13\%) questions had an average importance score lower than five. The three questions that ranked lowest in importance related to getting help with practical issues and carrying on with drinking alcohol or smoking. 
Table 2 Mean importance scores of questions in decreasing order of perceived importance ${ }^{a}$, and number of patients who considered that it should be avoided to answer the question or that the question is not applicable in the first weeks after (the likely) diagnosis ( $N=47$ patients)

\begin{tabular}{|c|c|c|c|c|c|}
\hline & & Mean & SD & Avoid & Not applicable \\
\hline 1 & How certain is it that there are no metastases? $?^{b, c}$ & 6.84 & .367 & 2 & 0 \\
\hline 2 & Can I still have surgery for my pancreatic cancer? $?^{\mathrm{b}, \mathrm{c}}$ & 6.80 & .405 & 1 & 6 \\
\hline 3 & What are my chances to be cured of pancreatic cancer? $?^{\mathrm{b}, \mathrm{c}}$ & 6.74 & .444 & 1 & 0 \\
\hline 4 & Which treatments can I have? $?^{\mathrm{b}, \mathrm{c}}$ & 6.72 & .454 & 1 & 2 \\
\hline 5 & If my pancreatic cancer is not treated, how fast will it then spread? ${ }^{\mathrm{b}, \mathrm{c}}$ & 6.66 & .708 & 0 & 7 \\
\hline 6 & If my pancreatic cancer is not treated, will I die from it? ${ }^{c}$ & 6.59 & 1.093 & 3 & 5 \\
\hline 7 & How does the treatment work? ${ }^{\mathrm{b}}$ & 6.58 & .621 & 1 & 1 \\
\hline 8 & What side effects could occur due to the treatment? & 6.57 & .655 & 0 & 1 \\
\hline 9 & What is the probability of survival after the surgery that I will have? & 6.56 & .590 & 1 & 3 \\
\hline 10 & Where in the pancreas is the tumour that I have? ${ }^{c}$ & 6.55 & 1.044 & 2 & 1 \\
\hline 11 & What can I do against the side effects that occur due to the treatment? & 6.52 & .505 & 0 & 1 \\
\hline 12 & How fast will treatment affect my pancreatic cancer? & 6.51 & .631 & 1 & 3 \\
\hline 13 & What can I do if I am in pain? ${ }^{b}$ & 6.49 & .547 & 0 & 0 \\
\hline 14 & How often do I need to undergo my treatment? ${ }^{\mathrm{b}}$ & 6.49 & .592 & 2 & 2 \\
\hline 15 & Is it safe to wait before starting the treatment? ${ }^{c}$ & 6.48 & .851 & 3 & 13 \\
\hline 16 & What is pancreatic cancer? & 6.48 & 1.045 & 1 & 2 \\
\hline 17 & If the treatment is successful, will the cancer come back? & 6.48 & 1.130 & 0 & 1 \\
\hline 18 & What can I do to slow down the disease process? & 6.47 & .667 & 0 & 4 \\
\hline 19 & Why is there such a waiting time before starting the treatment? ${ }^{c}$ & 6.45 & .888 & 2 & 14 \\
\hline 20 & If my treatment has no effect, what are my options then? & 6.45 & .959 & 1 & 6 \\
\hline 21 & How long does the treatment last? & 6.44 & .693 & 1 & 1 \\
\hline 22 & If my pancreatic cancer is not treated, how long will I live? & 6.44 & 1.141 & 2 & 4 \\
\hline 23 & What is usually the natural course of pancreatic cancer? ${ }^{b}$ & 6.41 & 1.187 & 2 & 0 \\
\hline 24 & Who carries out the treatment? & 6.39 & .784 & 2 & 1 \\
\hline 25 & Why is my diagnosis still uncertain? & 6.37 & .809 & 2 & 15 \\
\hline 26 & What are the long term effects of the treatment? & 6.33 & 1.212 & 0 & 1 \\
\hline 27 & If my diagnosis is still uncertain, how soon after surgery will it be certain? & 6.31 & 1.087 & 1 & 20 \\
\hline 28 & What is the pancreas? & 6.30 & .795 & 0 & 3 \\
\hline 29 & Do I need to be admitted to the hospital for treatment? & 6.28 & .751 & 2 & 5 \\
\hline 30 & Can I make the decision myself if I do not want to live further? & 6.28 & 1.255 & 5 & 3 \\
\hline 31 & Shall I see my doctor during treatment? & 6.26 & .875 & 1 & 3 \\
\hline 32 & Are there other options for treatment that have not been mentioned? & 6.26 & 1.197 & 1 & 3 \\
\hline 33 & Why are the tumour and possible metastases not monitored during treatment? & 6.21 & .927 & 3 & 11 \\
\hline 34 & If my pancreatic cancer is not treated, will it spread? & 6.21 & 1.373 & 4 & 14 \\
\hline 35 & Can I still eat everything? & 6.20 & .786 & 1 & 1 \\
\hline 36 & Where is the pancreas located? & 6.19 & .833 & 2 & 3 \\
\hline 37 & What particular qualifications does my doctor have to treat pancreatic cancer? & 6.18 & 1.352 & 2 & 1 \\
\hline 38 & Will I be in pain because of my pancreatic cancer? & 6.15 & 1.095 & 1 & 0 \\
\hline 39 & How long will it take until I will feel better again, after treatment? & 6.13 & 1.147 & 0 & 1 \\
\hline 40 & Where can I find a diet specifically aimed at pancreatic cancer? & 6.11 & 1.280 & 0 & 3 \\
\hline 41 & What can I do if I am tired? & 6.07 & 1.063 & 0 & 1 \\
\hline 42 & Where are the best doctors in the field of pancreatic cancer? & 6.02 & 1.475 & 3 & 2 \\
\hline 43 & Will the pancreatic cancer make me tired? & 5.98 & 1.327 & 0 & 0 \\
\hline 44 & How many patients with pancreatic cancer has my doctor treated? & 5.98 & 1.488 & 3 & 1 \\
\hline
\end{tabular}


Table 2 Mean importance scores of questions in decreasing order of perceived importance ${ }^{\text {a }}$, and number of patients who considered that it should be avoided to answer the question or that the question is not applicable in the first weeks after (the likely) diagnosis ( $N=47$ patients) (Continued)

\begin{tabular}{|c|c|c|c|c|c|}
\hline 45 & How many patients are cured with treatment? & 5.98 & 1.592 & 1 & 2 \\
\hline 46 & Why would I choose to have treatment, if I cannot be cured anymore? & 5.97 & 1.449 & 3 & 13 \\
\hline 47 & Should I actively search for the best possible medical care? & 5.95 & 1.393 & 3 & 6 \\
\hline 48 & Could it be harmful that radiation goes through other organs as well? & 5.93 & 1.412 & 1 & 17 \\
\hline 49 & In how many patients does the treatment not lead to improvement? & 5.93 & 1.555 & 2 & 4 \\
\hline 50 & If I don't want treatment now, can I still get treated when the cancer gets worse? & 5.91 & 1.111 & 2 & 11 \\
\hline 51 & For how long do I have pancreatic cancer? & 5.91 & 1.461 & 3 & 1 \\
\hline 52 & Are my children at risk of developing pancreatic cancer? & 5.89 & 1.624 & 2 & 7 \\
\hline 53 & If my pancreatic cancer is not treated, shall I be able to continue taking care of myself? & 5.86 & 1.113 & 2 & 17 \\
\hline 54 & If I decide to postpone treatment, is there still a chance that I will be cured? & 5.77 & 1.633 & 2 & 14 \\
\hline 55 & If I decide to postpone treatment, shall there be treatment later on that can help me? & 5.73 & 1.461 & 3 & 14 \\
\hline 56 & Why is it that my pancreatic cancer was not diagnosed earlier? & 5.69 & 1.689 & 3 & 2 \\
\hline 57 & Can I continue to take care of myself during the treatment? & 5.67 & 1.443 & 0 & 4 \\
\hline 58 & What can I do about my emotional problems? & 5.66 & 1.132 & 2 & 3 \\
\hline 59 & Where can my caregivers find (psychological) help? & 5.62 & 1.139 & 2 & 7 \\
\hline 60 & $\begin{array}{l}\text { If my diagnosis is still uncertain, what are my chances that I have surgery which turns } \\
\text { out to be unnecessary? }\end{array}$ & 5.55 & 1.731 & 1 & 25 \\
\hline 61 & What would you do in my circumstances, doctor? & 5.51 & 1.956 & 1 & 3 \\
\hline 62 & If my pancreatic cancer is not treated, will my social life be affected? & 5.50 & 1.482 & 1 & 12 \\
\hline 63 & How often does my type of pancreatic cancer occur? & 5.47 & 1.316 & 0 & 0 \\
\hline 64 & If I refuse treatment, will my doctor continue to take care of me? & 5.47 & 1.722 & 3 & 12 \\
\hline 65 & Do I differ from the average patient with pancreatic cancer? & 5.45 & 2.051 & 2 & 3 \\
\hline 66 & Will the treatment affect my social life? & 5.36 & 1.708 & 0 & 5 \\
\hline 67 & Where can I get a good second opinion? & 5.33 & 1.639 & 1 & 6 \\
\hline 68 & What If I am no longer around? & 5.30 & 1.762 & 5 & 5 \\
\hline 69 & How do I tell my children and/or grandchildren that I am sick? & 5.29 & 1.601 & 1 & 11 \\
\hline 70 & Am I allowed to refuse treatment? & 5.26 & 1.738 & 2 & 9 \\
\hline 71 & Are there complementary treatments (for example acupuncture) that could support my treatment? & 5.23 & 1.699 & 3 & 9 \\
\hline 72 & Can I ask for a second opinion? & 5.20 & 1.636 & 1 & 6 \\
\hline 73 & How many patients like me have decided to have this treatment? & 5.05 & 1.962 & 2 & 3 \\
\hline 74 & Will the treatment make me lose my hair? & 4.92 & 2.033 & 0 & 10 \\
\hline 75 & Can I still drive while I get treatment? & 4.87 & 1.866 & 0 & 8 \\
\hline 76 & Can I still travel while I get treatment? & 4.85 & 1.791 & 1 & 6 \\
\hline 77 & Did I get my pancreatic cancer because of my way of life? & 4.84 & 1.914 & 1 & 3 \\
\hline 78 & Why does this happen to me? & 4.67 & 2.149 & 3 & 2 \\
\hline 79 & Can I talk to someone who has taken the treatment already? & 4.65 & 1.837 & 3 & 1 \\
\hline 80 & If my pancreatic cancer is not treated, shall I be able to continue working as I am used to? & 4.54 & 2.085 & 1 & 22 \\
\hline 81 & Can I continue working as I am used to, during treatment? & 4.26 & 2.036 & 2 & 7 \\
\hline 82 & Where can I find help for practical issues, such as delegating my bank matters? & 3.55 & 2.010 & 1 & 17 \\
\hline 83 & Can I continue drinking alcohol? & 3.43 & 2.150 & 2 & 16 \\
\hline 84 & Can I continue smoking? & 2.93 & 2.463 & 2 & 30 \\
\hline
\end{tabular}

If two questions had the same mean importance score, the question with the smallest standard deviation was considered as more important ${ }^{\mathrm{b}}$ These questions were part of the top 10 most important questions on average in the subsample of patients who completed the survey within 46 days since diagnosis

'These questions were part of the top 10 most important questions on average in the subsample of patients who completed the survey later than 46 days after diagnosis 
In total, for $67 / 84(80 \%)$ questions at least one participant (Md = 1 participant, range, $1-5)$ indicated that the question should be avoided in the first weeks after diagnosis (Table 2). Almost half $(31 / 67,46 \%)$ of these were questions with a median importance score of six or higher. The two questions considered most often better to avoid (each by five participants, 11\%) related to the possibility of deciding to end one's life and the time after the patient has died. Twenty-seven (57\%) participants indicated that none of the questions should be avoided, 16 (34\%) indicated that the answer to one to seven questions should be avoided, and four (8\%) preferred that the answer to 10 or more questions would be avoided. The $17 / 84$ questions that were never marked as better to avoid, included questions on the disease (prevalence, disease progression), treatment (harms, disease recurrence after treatment, how long before feeling better after treatment, effects on social life, self-care and ability to drive), diet, pain and fatigue.

Further, for $77 / 84$ (92\%) questions at least one participant $(\mathrm{Md}=3.5$ participants, range, $1-30)$ considered that the question was not applicable in the first weeks after diagnosis (Table 2). Five (11\%) participants indicated that all questions were applicable, 21 (45\%) indicated that between one and nine questions were not applicable, and $21(45 \%)$ considered 10 or more questions not applicable in the first weeks after diagnosis. Of the eight questions considered to be not applicable by one-third or more of the participants, two questions related to still having an uncertain diagnosis. Other questions related to treatment outcomes (ability to take care of oneself, to continue working or the effects on one's social life), and future possibilities for treatment if treatment is postponed. The seven questions which all participants considered applicable partly overlapped with the questions that never should be avoided and related to the disease (prevalence, natural course, stage, prognosis) and quality of life (pain, fatigue).

\section{Discussion}

This study aimed to identify how important patients consider an extensive set of disease- and treatmentrelated questions in the first period after their (likely) diagnosis of pancreatic cancer. If we look at the number of questions, on average the patients considered a large number to be important or very important to be addressed in the first period after diagnosis. Per participant this number ranged from 25 to $99 \%$ of the questions offered for consideration. This finding is in line with other studies showing that individual patients can greatly differ in the amount of information they consider relevant and/or want to receive $[6,8,12]$. The amount of information desired could not be predicted based on patients' socio-demographic characteristics.
If we look at how important the 84 questions that we offered were considered to be, the results showed that overall, patients considered it important that about half of the questions were addressed. Of all questions, patients considered it most important that questions were addressed about the diagnosis, likelihood of cure, treatment options, harms and procedures, prognosis if the disease is not treated, and quality of life (pain, fatigue, diet). Addressing this need should help patients to gain a better understanding of their situation. Having a good understanding of one's disease, prospects, and possible (medical) interventions is the best place to start when decisions about one's care need to be made and is expected to support coping with illness. However, addressing all these topics may cause patients to feel overwhelmed by the amount of information they need to process. In order to make it easier for patients to process, retain and review the information they would like to receive, doctors may wish to write down key information during the consultation and give it to the patient as a memory aid. Notably, questions may not need to be answered in a single consultation nor necessarily during a consultation. That is, doctors may spread the information over more than one consultation and/or defer provision of information to other sources, such as other healthcare providers or paper-based or electronic information sources. In this manner, the consultation can be dedicated to the issues that patients and their healthcare providers consider most critical at that particular moment in time, and that require a conversation in person.

Most of the questions considered as important to address were marked by some patients as better to avoid or as not being applicable. These patients may have considered the question to be confronting or irrelevant (e.g., questions about the diagnosis in case the diagnosis is certain) or untimely (e.g., questions about end-oflife issues) at that stage of the illness trajectory. The number of questions that patients considered better to avoid was small, and many did not wish to avoid any question, suggesting that patients generally do not wish to evade confronting topics, even in the context of a cancer diagnosis with such a poor prognosis. Healthcare professionals should not avoid introducing difficult topics, should ask patients about what they want to know, be honest, and try to be responsive to patients' verbal and nonverbal cues. There were comparatively more patients that considered questions to be not applicable than better to avoid, part of which were also the questions considered as least important on average. For the questions relating to diagnosing the disease, this may be explained by the patients having already received a certain diagnosis. Further, it was notable that the questions considered as not applicable often related to the option of having no treatment or to postponing 
treatment, options that may not have occurred to some as viable or desirable options.

There are some limitations to this study. We asked participants to think back to the first weeks after diagnosis. There was large variability in number of days between receiving the diagnosis and completing the survey. Eight participants filled out the survey within 10 days of diagnosis. These patients had less opportunity to gain insight in what questions were most important to them in the first period after diagnosis. Three participants on the other hand completed the survey more than 6 months after diagnosis. This could have affected their importance ratings, although research has shown that people can vividly remember negative emotional experiences [14]. As reported, we did not find indications for an effect of time since diagnosis on number of questions considered as important, or rank order of most important questions. We further deployed a broad recruitment strategy, both through clinics and via the internet. Possibly, different strategies appeal to different types of patients, which may be a strength if it leads to higher uptake but also a limitation as we do not know how this may affect the results. As a proxy for recruitment, we assessed whether patients who completed the paper versus online version of the questionnaire significantly differed in gender, age, education, number of days since diagnosis and number of questions considered to be important or very important and found none. Finally, we offered a ready-made list of questions to participants, which may have inflated the importance that they assigned to questions. Future research could look at what questions patients come up with themselves, for example by asking patients to record the questions they actually have in the first weeks after diagnosis, and ask them to rate their importance.

\section{Conclusion}

Patients consider many questions important to be answered in the first period after the (likely) diagnosis of pancreatic cancer, especially about the disease (natural course, stage, cure), treatment harms and procedures), pain and fatigue. Individual patients may distinctly differ from one another in their information preferences and preferences may be difficult to estimate without explicitly discussing them. Patients do further not seem to wish to avoid hearing about confronting issues, even in the context of a cancer diagnosis with such a poor prognosis. In order to avoid information overload and confusion in patients, the results of this study can help doctors to prioritize the information that they address. It could be helpful to additionally offer patients information on paper or online for their future reference.

\section{Acknowledgements}

We would like to thank Petra Roza, Marieke de Vries, and the Maasstad

Ziekenhuis, Reinier de Graaf hospital, the Radboud University Medical Centre and the St Antonius Hospital, Nieuwegein for their support during this research and Judith Verhagen for her comments on an earlier draft of the paper.

\section{Funding}

This work was supported by the Lisa Waller Hayes Foundation. The Lisa Waller Hayes foundation had no influence on study design, data collection, data analysis and interpretation, or reporting of the results.

Availability of data and materials

Please contact author for data requests.

\section{Authors' contributions}

LJMRS interviewed the patients, performed the analysis and drafted the manuscript. CJP participated in the design of the study and data collection. RJS and AHP conceived of the study and participated in the coordination of the study. AHP helped to draft the manuscript. All authors read and approved the final manuscript.

Ethics approval and consent to participate

The study was approved by the Medical Ethical Committee of the Leiden University Medical Center (reference number P13.019).

Consent for publication

Not applicable.

\section{Competing interests}

The authors declare that they have no competing interests.

\section{Publisher's Note}

Springer Nature remains neutral with regard to jurisdictional claims in published maps and institutional affiliations.

\section{Author details}

'Department of Social Psychology, Tilburg University, Tilburg, the Netherlands. ${ }^{2}$ Department of Surgery, Erasmus University Medical Centre, Rotterdam, the Netherlands. ${ }^{3}$ Department of Surgery, Leiden University Medical Centre, Leiden, the Netherlands. ${ }^{4}$ Department of Medical Decision Making, Leiden University Medical Centre, P.O. Box 9600, Leiden 2300 RC, the Netherlands.

Received: 3 April 2017 Accepted: 19 July 2017

Published online: 18 September 2017

\section{References}

1. Rahib L, Smith BD, Aizenberg R, Rosenzweig AB, Fleshman JM, Matrisian LM. Projecting cancer incidence and deaths to 2030: the unexpected burden of thyroid, liver, and pancreas cancers in the United States. Cancer Res. 2014;74:2913-21.

2. Li C, Heidt DG, Dalerba P, Burant CF, Zhang L, Adsay V, Wicha M, Clarke MF, Simeone DM. Identification of pancreatic cancer stem cells. Cancer Res. 2007:67:1030-7.

3. Wray JC, Ahmad SA, Matthews JB, Lowy AM. Surgery for pancreatic cancer: recent controversies and current practice. Gastroenterology. 2005;128:1626-41.

4. Garcea G, Dennison AR, Pattenden CJ, Neal CP, Sutton CD, Berry DP. Survival following curative resection for pancreatic ductal adenocarcinoma. A systematic review of the literature. J Pancreas. 2008;9:99-132.

5. Graydon J, Galloway S, Palmer-Wickham S, Harrison D, Rich-Van der Bij L, West P, Burlein-Hall S, Evans-Boyden B. Information needs of women during early treatment for breast cancer. J Adv Nurs. 1997;26:59-64.

6. Feldman-Stewart D, Brundage MD, Hayter C, Groome P, Nickel JC, Downes $\mathrm{H}$, Mackillop WJ. What questions do patients with curable prostate cancer want answered? Med Decis Mak. 2000;20:7-19.

7. Millis ME, Sullivan $K$. The importance on information giving for patients newly diagnosed with cancer a review of the literature. J Clin Nurs. 1999:8:631-42

8. Leydon GM, Boulton M, Moynihan C, Jones A, Mossman J. Cancer patients' information needs and information seeking behavior: in-depth interview study. BMJ. 2000;320:903-13.

9. Wittman E, Beaton C, Lewis WG, Hopper AN, Zamawi F, Jackson C, Dave B Bowen R, Willacombe A, Blackshaw G, Crosby TDL. Comparison of patients' 
needs and doctors' perceptions of information requirements related to a diagnosis of oesophageal or gastric cancer. Eur J Cancer Care. 2011;20:187-95.

10. Husson O, Mols F, Van der Pol-Franse LV. The relation between information provision and health-related quality of life, anxiety and depression among cancer survivors: a systematic review. Ann Oncol. 2010;22:761-72.

11. Jefford M, Tattersall MHN. Informing and involving cancer patients in their own care. Lancet Oncol. 2002;3:629-37.

12. Zeguers M, De Haes HCJM, Zandbelt LC, Ter Hoeven CL, Franssen SJ, Geijsen DD, Koning CCE, Smets EMA. The information needs of new radiotherapy patients: how to measure? Do they want to know everything? And if not, why? Int J Radiat Oncol Biol Phys. 2012;82:418-24

13. Feldman-Stewart D, Brundage MD, Hayter C, Groome P, Nickel JC, Downes $\mathrm{H}$, Mackillop WJ. What prostate cancer patients should know: variation in professionals' opinions. Radiother Oncol. 1998;49:111-23.

14. Kim JS, Vossel G, Gamer M. Effects of emotional context on memory for details: the role of attention. PLoS One. 2013;8:e77405.

Submit your next manuscript to BioMed Central and we will help you at every step:

- We accept pre-submission inquiries

- Our selector tool helps you to find the most relevant journal

- We provide round the clock customer support

- Convenient online submission

- Thorough peer review

- Inclusion in PubMed and all major indexing services

- Maximum visibility for your research

Submit your manuscript at www.biomedcentral.com/submit
Biomed Central 